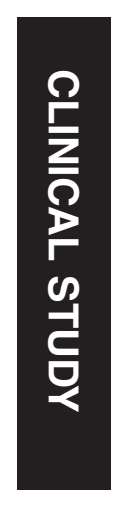

${ }^{1}$ Department of

Ophthalmology, University of North Carolina at Chapel Hill, Chapel Hill, NC, USA

${ }^{2}$ Glaucoma Associates of Texas, Dallas, TX, USA

${ }^{3}$ Duke University Eye Center, Durham, NC, USA

${ }^{4}$ Moorfields Eye Hospital, London, UK

${ }^{5}$ Johns Hopkins Bloomberg School of Public Health, Baltimore, MD, USA

${ }^{6}$ Peninsula Eye Hospital, Redcliffe, Queensland, Australia

${ }^{7}$ Bascom Palmer Eye Institute, University of Miami, Miami, FL, USA

${ }^{8}$ National Institute for Health Research, Biomedical Research Centre for Ophthalmology, Moorfields Eye Hospital, London, UK

Correspondence:

JC Mwanza, Department of Ophthalmology, University

of North Carolina at Chapel Hill, Chapel Hill, NC, 27599, USA

Tel: +1 919843 4917;

Fax: +1 9199661908 .

E-mail: jcmwanza@med.

unc.edu

Received: 26 June 2016 Accepted in revised form: 9 February 2017 Published online: 7 April 2017

\section{A comparison of cup-to-disc ratio estimates by fundus biomicroscopy and stereoscopic optic disc photography in the Tema Eye Survey}

JC Mwanza', DS Grover², DL Budenz'1, LW Herndon ${ }^{3}$, W Nolan ${ }^{4}$, J Whiteside-de Vos ${ }^{5}$, G Hay-Smith ${ }^{6}$, JR Bandi ${ }^{7}$, KA Bhansali', LA Forbes ${ }^{1}$, WJ Feuer ${ }^{7}$ and $K$ Barton $^{8}$

\begin{abstract}
Purpose To determine if there are systematic differences in cup-to-disc ratio (CDR) grading using fundus biomicroscopy compared to stereoscopic disc photograph reading. Methods The vertical cup-to-disc ratio (VCDR) and horizontal cup-to-disc ratio (HCDR) of 2200 eyes (testing set) were graded by glaucoma subspecialists through fundus biomicroscopy and by a reading center using stereoscopic disc photos. For validation, the glaucoma experts also estimated VCDR and HCDR using stereoscopic disc photos in a subset of $\mathbf{5 0 5}$ eyes that they had assessed biomicroscopically. Agreement between grading methods was assessed with Bland-Altman plots.
\end{abstract}

Results In both sets, photo reading tended to yield small CDRs marginally larger, but read large CDRs marginally smaller than fundus biomicroscopy. The mean differences in VCDR and HCDR were $0.006 \pm 0.18$ and $0.05 \pm 0.18$ (testing set), and $-0.053 \pm 0.23$ and $-0.028 \pm 0.21$ (validation set), respectively. The limits of agreement were $\sim 0.4$, which is twice as large as the cutoff of clinically significant CDR difference between methods. CDR estimates differed by 0.2 or more in $33.8-48.7 \%$ between methods.

Conclusions The differences in CDR estimates between fundus biomicroscopy and stereoscopic optic disc photo reading showed a wide variation, and reached clinically significance threshold in a large proportion of patients, suggesting a poor agreement. Thus, glaucoma should be monitored by comparing baseline and subsequent CDR estimates using the same method rather than comparing photographs to fundus biomicroscopy.
Eye (2017) 31, 1184-1190; doi:10.1038/eye.2017.50; published onlin 7 April 2017

Introduction

An increase in optic nerve head cupping, which is a sign of loss of neuroretinal rim tissue, is perhaps the most specific sign of glaucomatous optic neuropathy. ${ }^{1}$ However, detecting and documenting this change is challenging. Clinicians examine the optic disc using the direct ophthalmoscope, indirect ophthalmoscope or using the slit-lamp biomicroscope with an indirect condensing lens or direct contact lens. Fundus biomicroscopy is preferred to direct ophthalmoscopy because ascertainment of the cup-to-disc ratio (CDR) is more accurate under stereoscopic conditions. ${ }^{2,3}$ However, comparison of serial stereoscopic optic disc photographs is considered the gold standard for assessing glaucoma progression. ${ }^{4-7}$ Surprisingly, studies have shown that eye care providers do not routinely obtain stereoscopic disc photographs in practice. ${ }^{8,9}$ An intermediate option has been to compare the clinical examination of the optic disc to baseline stereoscopic photographs, as a good balance between diagnostic accuracy and cost effectiveness. ${ }^{10-12}$ However, the caveat is that clinical examination of the optic disc by fundus biomicroscopy can overestimate the amount of cupping when one compares what one sees clinically to evaluation of stereoscopic disc photographs. ${ }^{13}$ We have noticed in practice that optic disc cupping may be overestimated by fundus biomicroscopy compared to baseline stereoscopic photographs, which could result in falsely concluding that progressive cupping has occurred. The purpose of the current study is to 
determine if there are any systematic differences in the assessment of CDR using fundus biomicroscopy and stereoscopic disc photographs which could affect comparisons in the determination of progression.

\section{Materials and methods}

The stereoscopic optic disc photographs used in this study were collected as part of the Tema Eye Survey (TES), a population-based study conducted in Tema, Ghana, which was designed to determine the prevalence of blindness, visual impairment and eye diseases in an urban West African population of 5603 subjects aged 40 years and older. ${ }^{14,15}$ The TES received approval by the Institutional Review Boards of all US and UK investigators and Ethics Committee of the Ghana Ministry of Health. The study protocol adhered to the tenets of the Declaration of Helsinki and all participants signed an informed consent. Because the study design of the TES has been extensively detailed elsewhere, ${ }^{14,15}$ only the optic disc evaluation is presented herein.

\section{Cup-to-disc ratio estimation}

Participants in the TES underwent dilated assessment of the optic disc at the slit-lamp using a 90D (Volk Optical Inc., Mentor, OH, USA) condensing lens and simultaneous stereoscopic optic disc photography using a Nidek 3Dx camera (Nidek Co., Ltd., Tokyo, Japan) on the same day. During dilated fundus biomicroscopy, fellowship-trained glaucoma subspecialists estimated the vertical CDR (VCDR) and horizontal CDR (HCDR) in all study participants. The digital stereoscopic optic disc photos of the same subjects were submitted for VCDR and HCDR assessment at the reading center of the Moorfields Eye Hospital, London, UK. Before grading, the photographs were assessed on a large screen display for quality (clarity, adequacy of stereoscopy and field definition) and gradability. All gradable images of disc pairs were independently reviewed by two independent initial graders. The CDRs were determined by a more experienced third grader, who served as adjudicator when grading by the two initial graders was difficult. For this study a randomly selected subset of 2038 eyes of 1019 subjects was used for the comparison of CDR estimates by clinicians at the slit-lamp to those by the reading center readers. Of these, randomly selected optic disc photographs of 505 eyes (253 subjects) were submitted to the expert clinicians for CDR grading several years after the clinical examination was completed. The clinicians reviewed the image of the same subjects in whom they initially estimated the CDR through slit-lamp fundus biomicroscopy. Graders and expert clinicians used a hand-held stereoscope viewer (Screen-VU, Portland, OR, USA) to read the photographs.

\section{Statistical analysis}

The size of the disagreement between grading methods was calculated as their difference. A CDR difference equal to or greater than 0.2 between grading methods was considered as clinically significant disagreement. The proportion of eyes with a disagreement size of $0,0.1$, and $\geq 0.2$ was determined in the two sets. The mean CDRs of eyes with a CDR of 0.8 were compared between grading methods using the $t$-test because 0.7 is used as the upper limit of normal CDRs in studies in sub-Saharan Africa based on prior epidemiological studies. ${ }^{16-19}$ The incidence of visual field deficits increases significantly with VCDR greater than 0.7 in ocular hypertension and glaucoma, ${ }^{20}$ and based on our own findings that 0.725 was the 97.5 th percentile cutoff in the Tema population. ${ }^{14} \mathrm{Chi}$-square test was used to compare proportions. Bland and Altman plots $^{21}$ were used to assess the agreement between (1) CDR estimates by clinicians using slit-lamp fundus biomicroscopy and by the reading center using digital stereoscopic optic disc photographs and (2) CDR estimates obtained by fundus biomicroscopy and reading of stereoscopic optic disc photographs by the same clinicians. In both cases, the plots were obtained by regressing the difference in CDR (photo reading by the reading center or by clinicians minus fundus biomicroscopy) against the mean CDR ((photo reading by clinicians or reading center+fundus biomicroscopy)/2). A regression line was fitted to the scatterplot and the regression coefficient calculated to determine the presence or absence of a proportional bias in the scatterplot. The second comparison was performed to validate the findings of the first one. All statistical analyses were performed with SPSS version 23.0.

\section{Results}

\section{Demographic and clinical characteristics of study participants}

A total of 2038 eyes of 1019 subjects were included in this study. After excluding eyes with ungradable disc photos, data of 2032 eyes (1016 subjects) were available for statistical analysis. The mean age was $58.5 \pm 12.2$ years (range: 40-97 years). Eighty-two percent of the subjects had no ocular pathology and $11.7 \%$ had primary openangle glaucoma. Other types of glaucomas were seen in $2.8 \%$, ocular hypertension in $1.7 \%$ and non-glaucomatous optic neuropathies in $1.8 \%$ of the subjects.

\section{Size of disagreement and comparison between CDR estimation methods}

The size of the disagreement in VCDR and HCDR estimates made by fundus biomicroscopy compared to 
photo reading by the same clinicians and to photo reading by the reading center is shown in Table 1 . Of the eyes used to compare VCDR estimates $(n=2032)$ between fundus biomicroscopic and the reading center grading, the VCDR of 707 (34.8\%) eyes differed by $\geq 0.2$. Of the 505 eyes used to compare readings by fundus biomicroscopy to disc photo reading by the same clinicians, such a disagreement in VCDR was observed in 246 (48.7\%) eyes. The differences in proportions were statistically significant for both VCDR and HCDR (all $P<0.001$ ).

In the biomicroscopy $v$ s reading center and the biomicroscopy $v$ s photo reading by the same clinicians, 83 $(4.1 \%)$ and $37(7.3 \%)$ eyes, respectively, were graded as having a VCDR of 0.8 by the reading center and the by clinicians' photo reading $(P=0.002)$. Such proportions for HCDR were $4.4 \%(n=89)$ and $6.3 \%(n=32)$, respectively, $P=0.07$ (not in Table 2). The average VCDRs of these eyes by fundus biomicroscopy were $0.81 \pm 0.25$ and $0.84 \pm 0.17$; average HCDRs were $0.78 \pm 0.25$ and of $0.79 \pm 0.23$, respectively, all $P>0.05$ (Table 2 ).

\section{Bland-Altman analysis}

The mean differences in VCDR estimated by the reading center and fundus biomicroscopy was $0.006 \pm 0.184$ (limits of agreement (LOA): -0.36 to 0.37 ) (Figure 1a). The related regression coefficient was $-0.51(P<0.001)$. Such a difference in VCDR estimates between optic disc photography reading and fundus biomicroscopy by the clinicians was $-0.053 \pm 0.23$ (95\% LOA: -0.51 to 0.41 ), as shown in Figure 1c. The related regression coefficient was $-0.29(P<0.001)$. Values for HCDR are presented in Figure $1 \mathrm{~b}$ and $\mathrm{d}$. The negative regression slopes indicate that even though the average differences were zero, the reading center assessment of photos tended to read small CDRs marginally larger than the fundus biomicroscopy, but read large CDRs marginally smaller than the fundus biomicroscopy. This effect was also present when the expert clinicians reading photos compared to their own fundus biomicroscopy assessment, but was of smaller magnitude (shallower slopes).

\section{Discussion}

In the current study, we investigated the agreement in CDR estimates between fundus biomicroscopy and stereoscopic optic disc photograph reading in a population-based study in West Africa. The goal was to determine whether there is a systematic difference in CDR estimates between the two methods. There were no significant biases between the methods, but the LOA were about twice as large as the limit of significant clinical CDR difference between them. In addition, CDR estimates by the two methods differed by 0.2 or more in $33.5-48.7 \%$ of cases. Altogether, the results suggest poor agreement between fundus biomicroscopy and photo reading by either clinician experts or the reading center.

Although serial optic disc photography has been recommended as the preferred method for documenting optic disc changes over time, ${ }^{5,7,8}$ reports on the agreement between CDR estimation by specialized reading centers and fundus biomicroscopy by clinicians are still scarce. Only a limited number of studies have assessed the agreement of CDR estimates between stereo optic disc photography and fundus biomicroscopy. ${ }^{22-24}$ The current study is the first to compare CDR estimates between photo reading by a reading center and by fundus biomicroscopy, followed by the comparison of estimates by the same clinicians through fundus biomicroscopy and photo reading for validation purposes. While color stereoscopic optic disc photography or computerizedbased image analysis of the $\mathrm{ONH}$ is the best currently available methods for documenting ONH morphology in high-income countries, ${ }^{25}$ the situation is different in the

Table 2 Comparison of CDR of 0.8 graded by the reading center and glaucoma experts through photo reading with fundus biomicroscopy grades

\begin{tabular}{lccccc}
\hline$R C$ & $F B M$ & $P$ & $P R G$ & $F B M$ & $P$ \\
\hline VCDR $=0.8$ & $0.81 \pm 0.25$ & 0.76 & $\mathrm{VCDR}=0.8$ & $0.84 \pm 0.17$ & 0.16 \\
$\mathrm{HCDR}=0.8$ & $0.78 \pm 0.25$ & 0.44 & $\mathrm{HCDR}=0.8$ & $0.79 \pm 0.23$ & 0.88
\end{tabular}

Abbreviations: FBM, fundus biomicroscopy; HCDR, horizontal cup-todisc ratio; PRG, photo reading by glaucoma experts; $\mathrm{RC}$, reading center; VCDR, vertical cup-to-disc ratio.

Table 1 Size of disagreement in CDR estimates between photo reading and fundus biomicroscopy

\begin{tabular}{|c|c|c|c|c|c|c|}
\hline \multirow[t]{2}{*}{ Size of disagreement } & \multicolumn{3}{|c|}{$V C D R$} & \multicolumn{3}{|c|}{$H C D R$} \\
\hline & $F B M$ vs $R C$ & FBM vs $P R G$ & $\mathrm{P}$ & $F B M$ vs $R C$ & FBM vs $P R G$ & $\mathrm{P}$ \\
\hline 0 & $508(25.0 \%)$ & $87(17.2 \%)$ & $<0.001$ & $532(26.2 \%)$ & $99(19.6 \%)$ & 0.002 \\
\hline 0.1 & $817(40.2 \%)$ & $172(34.1 \%)$ & 0.011 & $811(40.0 \%)$ & $204(40.4 \%)$ & 0.86 \\
\hline$\geq 0.2$ & $707(34.8 \%)$ & $246(48.7 \%)$ & $<0.001$ & $687(33.8 \%)$ & $202(40.0 \%)$ & $<0.001$ \\
\hline
\end{tabular}

Abbreviations: FBM, fundus biomicroscopy; HCDR, horizontal cup-to-disc ratio; PRG, photo reading by glaucoma experts; RC, reading center; VCDR, vertical cup-to-disc ratio. 
a

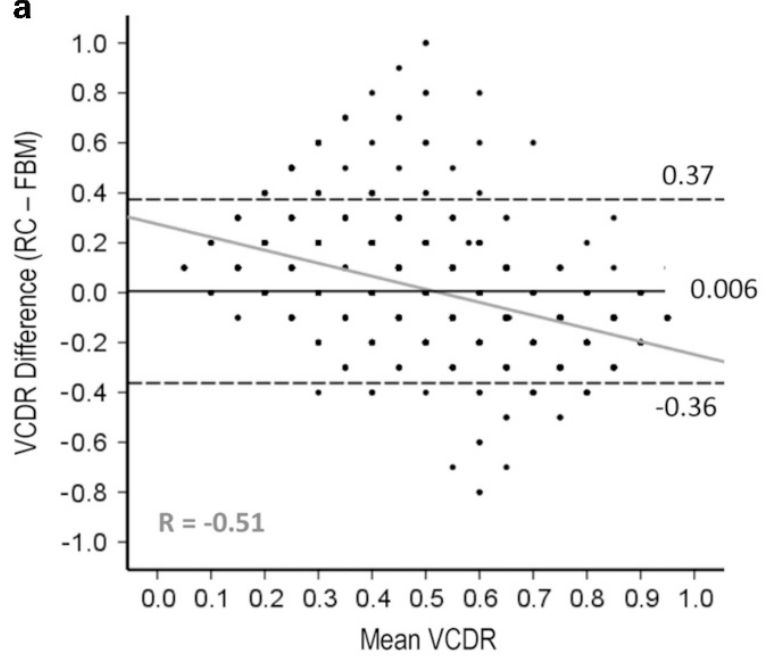

C

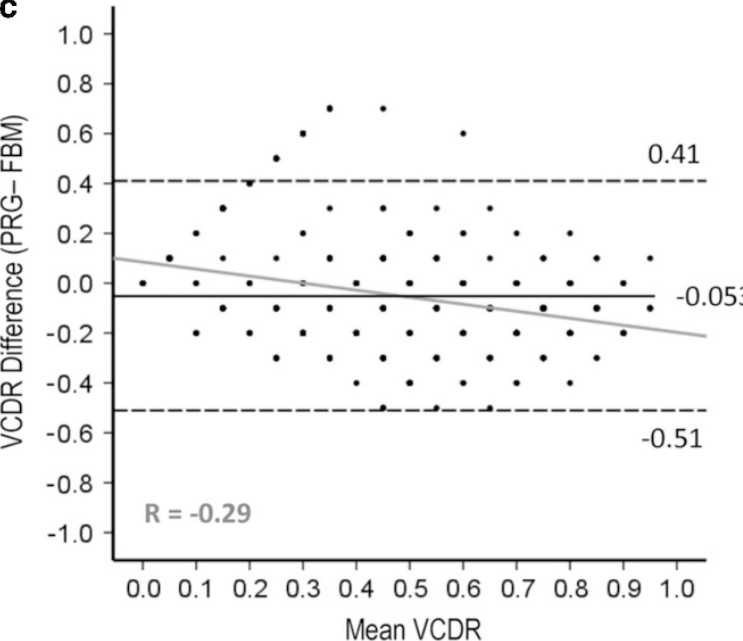

b

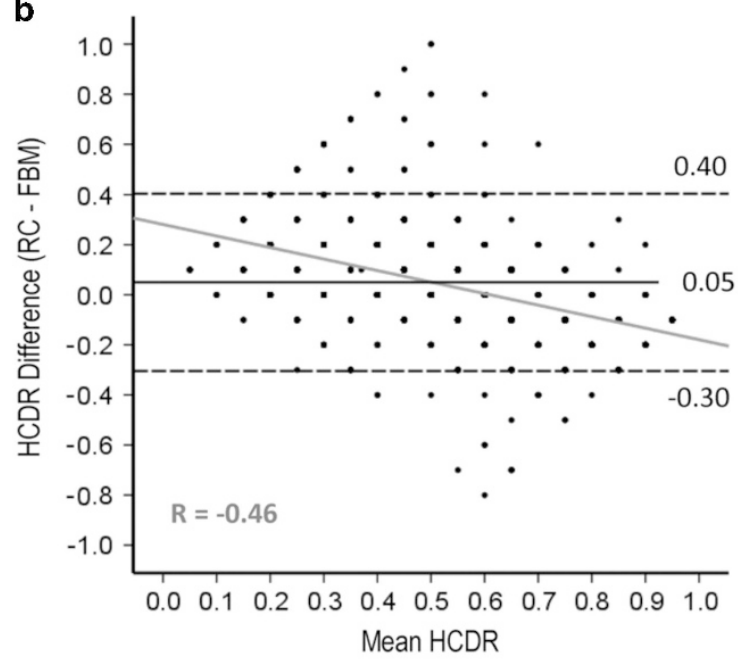

d

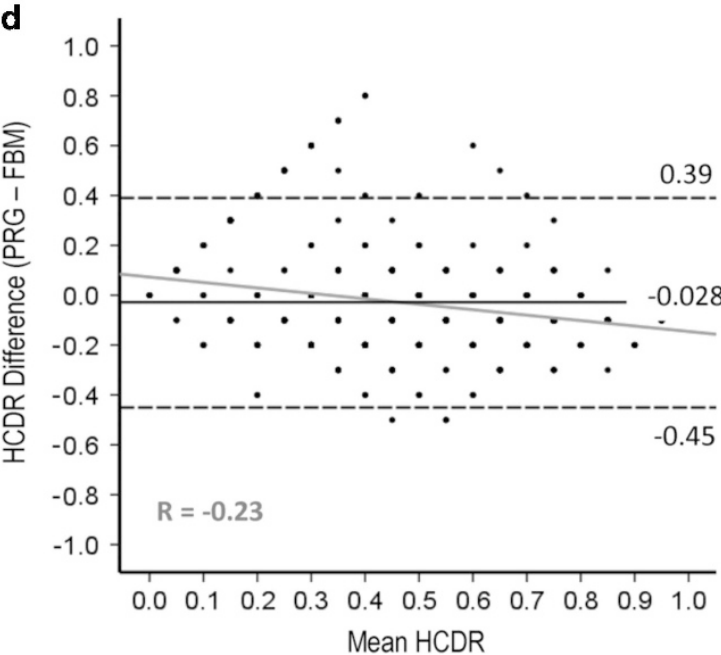

Figure 1 Bland-Altman plots of the agreement between the reading center (RC) and fundus biomicroscopy (FBM) in grading VCDR (a) and HCDR (b), and between photo reading by glaucoma experts (PRG) and fundus biomicroscopy in estimating VCDR (c) and HCDR (d). The horizontal plain line in the center of each plot represents the average difference in CDR estimated between the methods (RC or PRG-FBM), whereas the dotted lines indicate the upper and lower 95\% confidence intervals of the limits of agreement (LOA). The proportional bias in each plot in indicated by the significant slope of the gray line regressing the difference in CDR against the average CDR ((RC or PRG+FBM)/2).

developing world, where clinical fundus examination is the method widely used.

This study found larger VCDR and HCDR biomicroscopically compared with stereoscopic digital image reading by the clinicians. This finding is in line with that of a previous study. ${ }^{2}$ Kyari and Gilbert ${ }^{24}$ recently investigated the agreement of fundus biomicroscopic VCDR with digital monoscopic optic disc photo reading by the Moorfields Disc Reading Center. They analyzed 1696 eyes of 848 Nigerians aged 40 years and older in a population-based study. Their finding contrasted with ours in that the mean VCDR was significantly larger by photo reading than fundus biomicroscopy. Their results also indicated that VCDR from the two methods differed by 0.2 or less in $94 \%$, which is comparable to $88.1 \%$ in our study. We found that the proportion of VCDR and HCDR that differed by $\geq 0.2$ ranged between 33.8 and $40 \%$ in both comparisons, suggesting that one should be cautious when trying to use interchangeably biomicroscopic and photo reading estimates to assess glaucoma progression. In addition, the SD on the order of 0.2 (Table 2) are large enough to be concerning, meaning that a comparison of baseline CDR estimated by photo reading to subsequent fundus biomicroscopy CDR or vice-versa may result in substantial number of cases 'progressing', as well as cases with 
apparent 'improvements' with no actual change. While this is the first time such high proportions of disagreement are reported, an earlier study by Hrynchak et $a l^{26}$ that compared CDRs using stereoscopic biomicroscopy and digital photos found proportions of $5 \%$ for HCDR and $17.5 \%$ for VCDR for one clinician and 12.5 and $25 \%$ respectively for another, which confirms the interobserver variability using both methods. However, that study included only normal subjects and used nonstereoscopic photos.

In both cases Bland-Altman plots also showed a substantial variability in CDR between fundus biomicroscopy and photo reading. The LOA were roughly in the order of 0.4 , which is larger considering 0.2 is a clinically substantial difference, suggesting a poor agreement despite the mean differences being close to zero. This probably is related to the challenge of accurately delineating the optic disc with either of these subjective methods, resulting in differing measurements. The results of the photo reading $v$ s fundus biomicroscopy by the same clinicians confirmed the reading center $v s$ fundus biomicroscopy findings in terms of agreement, suggesting a systematic difference between the two CDR estimation methods. This is in line with what Kyari and Gilbert reported. ${ }^{24}$ As said previously, the clinical corollary of this finding is that the baseline and follow-up CDR assessment should be made using the same method rather than comparing photographs to fundus biomicroscopy. While the use of optic disc photography with analysis by a reading center is ideal for glaucoma prevalence studies as recommended by others, ${ }^{24}$ it is not currently applicable in this setting without external assistance for the above-mentioned reasons and the lack of reading centers. Thus, the best option for glaucoma prevalence studies and diagnosing glaucoma progression in sub-Saharan Africa using CDR at present may be to compare baseline and follow-up fundus biomicroscopy estimates. Conversion to and proficiency in estimation of CDR by stereoscopic fundus biomicroscopy and photography may require additional training of general ophthalmologists in this setting. Several factors justify this choice, including (1) the high cost associated with serial disc photos that might discourage patients from attending follow-up visits, (2) the lack of electronic medical record and storage systems and (3) the rarity of modern fundus cameras devices at present, particularly in governmentrun health care facilities where the large majority of the population seeks care. Fundus biomicroscopy should be preferred over direct ophthalmoscopy, which is the current most widely used method in sub-Saharan Africa, because the former yields more accurate and less variable estimates than the latter. Indeed, viewing the optic disc in a two-dimensional fashion can result in estimate inaccuracies and both lower specificity and sensitivity for diagnosing glaucoma, as the quality and size of the fundus image is highly dependent on the subject's refraction and the clarity of ocular media. ${ }^{27}$ On the contrary, fundus biomicroscopy with a high-powered condensing lens yields a stereoscopic image that is less affected by refraction and ocular media opacities. Dhar et $a l^{28}$ performed a comparative study of CDR estimated with fundus biomicroscopy, stereoscopic fundus photography with a 90D lens, and time domain optical coherence tomography in small sample size of 15 subjects with and 15 others without glaucoma. They only reported the Pearson correlation coefficient between pairs of methods, which does not indicate whether there is a systematic bias or not, but rather merely indicates how the values scatter around the line of best fit, regardless of its slope. ${ }^{29}$

The strengths of our study include its large sample size and its population-based design that allowed us to estimate the agreement between methods of CDR estimation across a wide range of optic disc features in the general population. For this reason, the findings of this study may not be directly comparable to those obtained from glaucoma clinic patients who are often referred either because of suspected large cups with no visual field defects or other risk factors (among whom most will be normal subjects) or because of suspicious cups in addition to other glaucoma risk factors. Another strength is the use of stereoscopic rather than monoscopic photos. Prior studies have shown that assessment of optic disc using stereoscopic images yields excellent quality, reproducibility and better interobserver agreement compared with monoscopic images. ${ }^{30,31}$

We understand that it would have been informative to know the influence of optic disc size on the agreement in estimating CDR because the agreement in VCDR estimates between stereoscopic optic disc photo reading and fundus biomicroscopy is better for larger than for smaller discs, regardless of whether the eyes are normal, glaucomatous or only suspected of having glaucoma. ${ }^{26}$ The results of this study do not imply that glaucoma monitoring should be decided based on CDR assessment alone, because of its limited value in identifying glaucomatous discs as a result of the large variation in optic disc anatomy in normal subjects. Other important funduscopic findings such as the neuroretinal rim characteristics, the presence or absence of a retinal nerve fiber layer defect or optic disc hemorrhage, and the size and type of peripapillary atrophy should be considered.

In conclusion, the poor agreement between stereoscopic disc photo reading and fundus biomicroscopy in estimating VCDR and HCDR suggests that CDR should be monitored by comparing serial estimates using the same method. In settings with limited resources such as sub-Saharan Africa, fundus biomicroscopy might be 
better than the current, widely-used method of evaluating changes in the optic nerve with the direct ophthalmoscope.

\section{Summary}

\section{What was known before}

- CDR estimates are known to be variable, when assessed with fundus biomicroscopy or stereoscopic optic disc photos. Fundus biomicroscopy can overestimate the CDR compared to stereoscopic photo reading. Color stereoscopic optic disc photography or computerized image analysis of the $\mathrm{ONH}$ is the recommended method for documenting glaucomatous change to the $\mathrm{ONH}$ morphology.

\section{What this study adds}

- Fundus biomicroscopy and stereoscopic optic disc photo estimates of CDR disagreed in a large proportion of patients.

- This study does not support the concept of comparing fundus biomicroscopy with optic disc photo reading for monitoring glaucoma over time. Instead, glaucoma should be monitored by comparing baseline and subsequent CDR estimates using the same method. In low-resource settings such as sub-Saharan Africa, comparing baseline and subsequent fundus biomicroscopy CDR estimates would be the preferred method.

\section{Conflict of interest}

The authors declare no conflict of interest.

\section{Acknowledgements}

We wish to thank Elizabeth Mathenge for helping with data cleaning. Supported by Research to Prevent Blindness, New York, NY, USA.

\section{References}

1 Anderson DR. What happens to the optic disc and retina in glaucoma? Ophthalmology 1983; 90: 766-770.

2 Varma R, Steinmann WC, Scott IU. Expert agreement in evaluating the optic disc for glaucoma. Ophthalmology 1992; 99: 215-221.

3 Watkins R, Panchal L, Uddin J, Gunvant P. Vertical cup-to-disc ratio: agreement between direct ophthalmoscopic estimation, fundus biomicroscopic estimation, and scanning laser ophthalmoscopic measurement. Optom Vis Sci 2003; 80: 454-459.

4 Kass MA, Heuer DK, Higginbotham EJ, Johnson CA, Keltner JL, Miller JP et al. The ocular hypertension treatment study: a randomized trial determines that topical ocular hypotensive medication delays or prevents the onset of primary open-angle glaucoma. Arch Ophthalmol 2002; 120: 701-713.
5 Leske MC, Heijl A, Hyman L, Bengtsson B. Early manifest glaucoma trial: design and baseline data. Ophthalmology 1999; 106: 2144-2153.

6 Medeiros FA, Alencar LM, Zangwill LM, Bowd C, Sample PA, Weinreb RN. Prediction of functional loss in glaucoma from progressive optic disc damage. Arch Ophthalmol 2009; 127: 1250-1256.

7 Nicolela MT, McCormick TA, Drance SM, Ferrier SN, LeBlanc RP, Chauhan BC. Visual field and optic disc progression in patients with different types of optic disc damage: a longitudinal prospective study. Ophthalmology 2003; 110: 2178-2184.

8 Fremont AM, Lee PP, Mangione CM, Kapur K, Adams JL, Wickstrom SL et al. Patterns of care for open-angle glaucoma in managed care. Arch Ophthalmol 2003; 121: 777-783.

9 Hertzog LH, Albrecht KG, LaBree L, Lee PP. Glaucoma care and conformance with preferred practice patterns. Examination of the private, community-based ophthalmologist. Ophthalmology 1996; 103: 1009-1013.

10 Budenz DL, Palmberg PF. Current cost-effective work-up of the glaucoma suspect. Curr Opin Ophthalmol 1996; 7: 59-64.

11 Ederer F, Gaasterland DE, Sullivan EK. The Advanced Glaucoma Intervention Study (AGIS): 1. Study design and methods and baseline characteristics of study patients. Control Clin Trials 1994; 15: 299-325.

12 Gaasterland DE, Blackwell B, Dally LG, Caprioli J, Katz LJ, Ederer F. The Advanced Glaucoma Intervention Study (AGIS): 10. Variability among academic glaucoma subspecialists in assessing optic disc notching. Trans Am Ophthalmol Soc 2001; 99: 177-184.

13 Tielsch JM, Katz J, Quigley HA, Miller NR, Sommer A. Intraobserver and interobserver agreement in measurement of optic disc characteristics. Ophthalmology 1988; 95: 350-356.

14 Budenz DL, Bandi JR, Barton K, Nolan W, Herndon L, Whiteside-de Vos J et al. Blindness and visual impairment in an urban West African population: the Tema Eye Survey. Ophthalmology 2012; 119: 1744-1753.

15 Budenz DL, Barton K, Whiteside-de Vos J, Schiffman J, Bandi J, Nolan W et al. Prevalence of glaucoma in an urban West African population: the Tema Eye Survey. JAMA Ophthalmol 2013; 131: 651-658.

16 Bastawrous A, Giardini ME, Bolster NM, Peto T, Shah N, Livingstone IA et al. Clinical validation of a smartphonebased adapter for optic disc imaging in Kenya. JAMA Ophthalmol 2016; 134: 151-158.

17 Buhrmann RR, Quigley HA, Barron Y, West SK, Oliva MS, Mmbaga BB. Prevalence of glaucoma in a rural East African population. Invest Ophthalmol Vis Sci 2000; 41: 40-48.

18 Foster PJ, Buhrmann R, Quigley HA, Johnson GJ. The definition and classification of glaucoma in prevalence surveys. Br J Ophthalmol 2002; 86: 238-242.

19 Rotchford AP, Kirwan JF, Muller MA, Johnson GJ, Roux P. Temba glaucoma study: a population-based cross-sectional survey in urban South Africa. Ophthalmology 2003; 110: 376-382.

20 Gloster J. Quantitative relationship between cupping of the optic disc and visual field loss in chronic simple glaucoma. Br J Ophthalmol 1978; 62: 665-669.

21 Bland JM, Altman DG. Statistical methods for assessing agreement between two methods of clinical measurement. Lancet 1986; 1: 307-310.

22 Durmus M, Karadag R, Erdurmus M, Totan Y, Feyzi Hepsen I. Assessment of cup-to-disc ratio with slit-lamp funduscopy, Heidelberg Retina Tomography II, and stereoscopic photos. Eur J Ophthalmol 2009; 19: 55-60. 
23 Jayasundera T, Danesh-Meyer HV, Donaldson M, Gamble G. Agreement between stereoscopic photographs, clinical assessment, Heidelberg retina tomograph and digital stereoscopic optic disc camera in estimating vertical cup:disc ratio. Clin Experiment Ophthalmol 2005; 33: 259-263.

24 Kyari F, Gilbert C. Agreement in measurement of optic cup-to-disc ratio with stereo biomicroscope funduscopy and digital image analysis: results from the Nigeria National Blindness and Visual Impairment Survey. Ophthalmic Epidemiol 2017; 24: 57-62.

25 Prum Jr BE, Rosenberg LF, Gedde SJ, Mansberger SL, Stein JD, Moroi SE et al. Primary open-angle glaucoma preferred practice pattern (R) guidelines. Ophthalmology 2016; 123: P41-P111.

26 Hrynchak P, Hutchings N, Jones D, Simpson T. A comparison of cup-to-disc ratio evaluation in normal subjects using stereo biomicroscopy and digital imaging of the optic nerve head. Ophthalmic Physiol Opt 2003; 23: 51-59.
27 Harper R, Reeves B. The sensitivity and specificity of direct ophthalmoscopic optic disc assessment in screening for glaucoma: a multivariate analysis. Graefes Arch Clin Exp Ophthalmol 2000; 238: 949-955.

28 Dhar M, Jayachandran I, Raju B, Deepa PA. Comparison of results of optic disc analysis using stereoscopic biomicroscopy, stereo fundus photography and optical coherence tomography. Kerala J Ophthalmol 2007; 19: 276-281.

29 Ludbrook J. Linear regression analysis for comparing two measurers or methods of measurement: but which regression? Clin Exp Pharmacol Physiol 2010; 37: 692-699.

30 Morgan JE, Sheen NJ, North RV, Choong Y, Ansari E. Digital imaging of the optic nerve head: monoscopic and stereoscopic analysis. Br J Ophthalmol 2005; 89: 879-884.

31 Parkin B, Shuttleworth G, Costen M, Davison C. A comparison of stereoscopic and monoscopic evaluation of optic disc topography using a digital optic disc stereo camera. $\mathrm{Br} \mathrm{J}$ Ophthalmol 2001; 85: 1347-1351. 